\title{
Acoustic radiation by vortex induced flexible wall vibration
}

\author{
S. K. Tang ${ }^{\text {a) }}$ \\ Department of Building Services Engineering, The Hong Kong Polytechnic University, Hung Hom, \\ Hong Kong, Hong Kong nil Hong Kong \\ R. C. K. Leung and R. M. C. So \\ Department of Mechanical Engineering, The Hong Kong Polytechnic University, Hong Kong, \\ People's Republic of China \\ K. M. Lam \\ Department of Civil Engineering, The University of Hong Kong, Hong Kong, People's Republic of China
}

(Received 18 February 2005; revised 15 May 2005; accepted 7 July 2005)

\begin{abstract}
Sound radiation due to unsteady interaction between an inviscid vortex (which models a turbulent eddy) and a finite length flexible boundary in a two-dimensional space is studied using potential theory and the matched asymptotic expansion technique. The Mach number of the vortex propagation is kept below 0.15 . Results suggest that the monopole field created by the volumetric flow induced by the vibrating flexible boundary dominates the overall acoustic power radiation. The longitudinal dipole directly due to the transverse vortex acceleration is only important when the vortex is moving over the flexible boundary. The longitudinal dipole resulting from the boundary vibration gains slightly in importance in the strong vortex case, but the corresponding transverse dipole remains negligible for the cases considered in the present study. The two longitudinal dipoles give rise to biased radiation directivities on both sides of the flexible boundary. (C) 2005 Acoustical Society of America. [DOI: 10.1121/1.2011127]
\end{abstract}

PACS number(s): 43.28.Ra, 43.40.Rj, 43.50.Nm [GL]

Pages: 2182-2189

\section{INTRODUCTION}

Air conditioning and ventilation system is a major noise source in any commercial building. The associated air ductwork conveys turbulent flows from the fans to the interior workplaces. The ducted elements will affect the turbulence thus generated and the propagation of noise. Understanding the flow-structure-acoustics interactions is therefore of prime importance in modern building noise control.

The study of the interaction between structural vibration and acoustics has attracted the attention of many researchers in the past few decades. For instance, Frendi and Robinson, ${ }^{1}$ Cummings, ${ }^{2}$ and $\mathrm{Huang}^{3}$ studied the duct wall vibration induced by a downstream traveling plane acoustic wave in the absence of a mean flow. Blake ${ }^{4}$ and Leppington et al. ${ }^{5}$ examined the acoustic radiation by vibrating panels, while $\mathrm{Wu}$ and Maestrello, ${ }^{6}$ Dowell, ${ }^{7}$ and Howe ${ }^{8}$ investigated the acoustic responses of a plate subject to a distributed surface pressure loading (which is used to simulate the effects of a turbulent boundary layer). The scattering of sound by a panel in the absence of a flow was also studied by many researchers, for instance, Abrahams ${ }^{9}$ and Leppington. ${ }^{10}$ In addition, Graham $^{11}$ showed theoretically that the curvature of a panel has significant influence on its sound radiation. Ffowcs Williams and Lovely ${ }^{12}$ proved that the presence of a steady low Mach number mean flow tends to amplify the sound generated by surface vibration. Davies ${ }^{13}$ studied the overall sound power radiated from panels excited by a turbulent

\footnotetext{
${ }^{a)}$ Electronic mail: besktang@ @olyu.edu.hk
}

boundary layer and compared his predictions with experimental results. However, details of the flow-structureacoustics interactions were not presented.

The turbulent air flow inside air conditioning ductwork induces pressure fluctuations on the duct walls, resulting in the vibration of the latter which in turn radiates sound to both the duct interior and the external environment (breakout noise). ${ }^{14}$ However, corresponding studies on the effect of flow turbulence on noise generated by flow-induced structural vibrations is rarely found. The effect of turbulent flow on the effectiveness of the duct noise control using flexible membranes proposed by Huang ${ }^{3}$ is also not clear.

The air flow inside any ductwork in a building is of very low Mach number $(\leqslant 0.1)$. The acoustic analogy ${ }^{15,16}$ works well in the estimation of the aerodynamic sound production at such low Mach number condition provided that the flow field and the associated solid surface vibration are precisely known. However, the exact coupling between the turbulent flow with the duct wall cannot be analytically handled such that one has to turn to computational technique whose accuracy might still be subject to query. The computations are also very resource demanding. Vortices, though, are drastic simplifications of the real flows, and have been adapted extensively in the study of sound generation by turbulent flows in the presence of a solid object because of their simplicity such that analytical solutions can be obtained in some cases. Typical examples of these studies include Howe, ${ }^{17}$ Obermeier $^{18}$ and the more recent work of Leung and So ${ }^{19}$ and Tang and Lau. ${ }^{20}$

In the present investigation, a vortex is chosen to represent a turbulent eddy, and the motion of a flexible boundary 


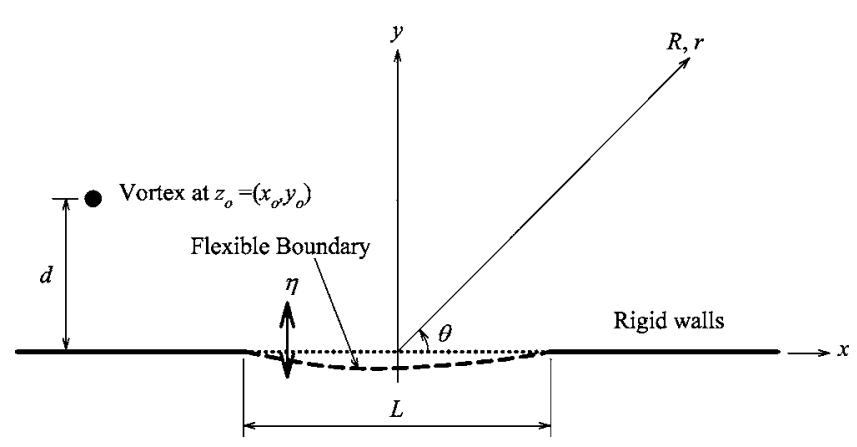

FIG. 1. Schematic of the theoretical model.

in an otherwise rigid plane under the actions of this vortex is calculated. The sound so radiated into the far field on both sides of this boundary will be analyzed in detail. The effects of the mechanical properties of the boundary will also be examined. It is hoped that the present results can provide information for improved duct noise control design and contribute to the fundamental understanding of the flowstructure-acoustic interaction.

\section{THEORETICAL MODEL}

\section{A. Motions of the vortex and the flexible boundary}

Since air viscosity and compressibility are of secondary importance for low Mach number flow noise generation, ${ }^{21}$ an incompressible inviscid vortex model is adopted in the present study. Figure 1 illustrates the schematic of the present study model. A vortex of strength (circulation) $\Gamma$ is initially located far upstream of the flexible boundary of length $L$ at a distance $d$ above the rigid plane. The initial speed of the vortex, $U_{i}$, therefore equals $\Gamma /(4 \pi d)^{22}$ and is in a direction parallel to the rigid plane. The flexible boundary is at rest initially. The movement of the vortex creates a time varying fluid pressure on this boundary, causing it to vibrate and this vibration eventually gives rise to a fluctuating velocity field, which affects the motion of the vortex.

It is assumed that the vibration amplitude of the flexible boundary, $\eta(x, \tau)$, is small compared to $d$ (and $y_{0}$ ) and this boundary is modeled as a rigid surface with distributed fluctuating velocity $v(x, \tau)(=\partial \eta / \partial \tau)$, where $\tau$ denotes the near field time. The edges of this flexible boundary are kept fixed and thus $\eta(-L / 2, \tau) / d=\eta(L / 2, \tau) / d=0$. The induced velocity at the vortex position $\left(x_{0}, y_{0}\right)$ due to the boundary vibration, $V_{i}$, can be determined using potential theory ${ }^{22}$ as

$$
V_{i}=\frac{\hat{x}}{\pi} \int_{-L / 2}^{L / 2} \frac{v\left(x_{0}-x\right)}{\left(x_{0}-x\right)^{2}+y_{0}^{2}} d x+\frac{\hat{y}}{\pi} \int_{-L / 2}^{L / 2} \frac{v y_{0}}{\left(x_{0}-x\right)^{2}+y_{0}^{2}} d x,
$$

where the caret denotes unit vector in the direction indicated. The vortex velocity, $V$, is thus

$$
V=\frac{\Gamma}{4 \pi y_{0}} \hat{x}+V_{i}
$$

Owing to the very thin flexible duct wall compared to its length in practice, the effect of the bending stiffness is usually insignificant when compared to that of the tension within the wall. For a wall thickness of $0.5 \mathrm{~mm}$, which is the usual value for a flow duct, and a duct length of say $10 \mathrm{~mm}$, the error of neglecting the bending term is less than $0.3 \%$. The longer the duct length, the smaller this error will be. Therefore, for simplicity, the time variations of $v(x, \tau)$ are obtained by solving the dynamic equation governing the forced vibration of a membrane, ${ }^{3}$ or

$$
M \frac{\partial^{2} \eta}{\partial \tau^{2}}=T \frac{\partial^{2} \eta}{\partial x^{2}}-D \frac{\partial \eta}{\partial \tau}-\Delta p
$$

where $M, T$, and $D$ denote the surface density, the tension per unit spanwise length, and the damping coefficient of the membrane, respectively, and $\Delta p$ represents the differential air pressure between the upper and lower side of the membrane, which is

$$
\begin{aligned}
\Delta p= & -\rho \frac{\partial \phi}{\partial \tau}=\frac{\rho \Gamma}{2 \pi} \frac{\partial}{\partial \tau} \tan ^{-1}\left[\frac{2 y_{0}\left(x-x_{0}\right)}{\left(x-x_{0}\right)^{2}+\left(\eta^{2}-y_{0}^{2}\right)}\right] \\
& -\frac{2 \rho}{\pi} \frac{\partial}{\partial \tau} \int_{-L / 2}^{L / 2} \frac{\partial \eta^{\prime}}{\partial \tau} \log \sqrt{\left(x-x^{\prime}\right)^{2}+\left(\eta-\eta^{\prime}\right)^{2}} d x^{\prime},
\end{aligned}
$$

where the prime denotes a quantity along the flexible boundary. For small magnitude low frequency boundary vibration, contribution from the second term on the right-hand side of Eq. (4), which represents a kind of fluid loading, ${ }^{23}$ is weak as long as the vortex is in the proximity of the boundary. This is the case of the present study.

The motion of the vortex and the distributed velocity $v(x, \tau)$ can be updated by solving Eqs. (2)-(4) together with the instantaneous profile $\eta(x, \tau)$ using a fourth order RungeKutta time integration scheme. The initial conditions are $V$ $=U_{i}, \eta(x, 0) / d=0$ and $v(x, 0) / U_{i}=0$. One can observe that the present approach is very different from that adopted by Walker et $a l .{ }^{24}$ in which the vortex is always moving parallel to the wall.

\section{B. Acoustic far field}

The vibration of the flexible boundary and the unsteady motion of the vortex results in acoustic radiation on both sides of the boundary. At distance far away from the vortex and the flexible boundary, the fluid is at rest and the corresponding acoustic potential in frequency domain, $\phi_{0}$, is given by the solution of the Helmholtz equation,

$$
\nabla^{2} \phi_{0}+(\omega / c)^{2} \phi_{0}=0
$$

where $\omega$ denotes the angular velocity and $c$ the ambient speed of sound. In two-dimensional space, the outgoing wave solution of Eq. (5) at the radial distance $r$ is ${ }^{25}$

$$
\phi_{0}(r, \theta, \omega)=\sum_{\alpha} A_{\alpha} H_{\alpha}^{(1)}(\omega r / c) e^{i \alpha \theta},
$$

where $H_{\alpha}^{(1)}$ is the $\alpha$ th order Hankel function of the first kind and $A_{\alpha}$ is a term to be determined. One way to find $\alpha$ and $A_{\alpha}$ is by the method of matched asymptotic expansion as in Obermeier ${ }^{18}$ and Tang and Lau. ${ }^{20}$ The inner solution of the outer wave potential [Eq. (6)] at low frequency is 
matched with the outer solution of the inner incompressible near field potential.

\section{1. $y / d \rightarrow \infty$}

This is the region in which the sound is created by the vibrating flexible boundary alone. The fluid potential at a large distance $R$ in an angular direction $\theta$ from the center of the flexible boundary is

$$
\begin{aligned}
\phi= & -\frac{1}{\pi} \int_{-L / 2}^{L / 2} v \log \sqrt{(R \cos \theta-x)^{2}+(R \sin \theta-\eta)^{2}} d x \\
\approx & -\frac{1}{\pi} \log R \int_{-L / 2}^{L / 2} v d x \\
& +\frac{1}{\pi R} \int_{-L / 2}^{L / 2} v(x \cos \theta+\eta \sin \theta) d x .
\end{aligned}
$$

The properties of the Hankel functions ${ }^{25}$ and Eq. (7) suggests that the far field potential is

$$
\begin{aligned}
\phi_{0}(r, \theta, \omega)= & A_{0} H_{0}^{(1)}(\omega r / c) \\
& +H_{1}^{(1)}(\omega r / c)\left(A_{1} \cos \theta+B_{1} \sin \theta\right),
\end{aligned}
$$

where

$$
\begin{aligned}
& A_{0}=\frac{i}{2}\left[\int_{-L / 2}^{L / 2} v d x\right]^{F}, \quad A_{1}=\frac{i \omega}{2 c}\left\{\left[\int_{-L / 2}^{L / 2} v x d x\right]^{F}\right\}, \\
& B_{1}=\frac{i \omega}{2 c}\left[\int_{-L / 2}^{L / 2} v \eta d x\right]^{F}
\end{aligned}
$$

and []$^{F}$ denote the Fourier transform with respect to time. When $r \rightarrow \infty$,

$$
\begin{aligned}
\phi_{0}(r, \theta, \omega) \approx & \sqrt{\frac{2 c}{\pi \omega r}} e^{i \omega r / c}\left[A_{0} e^{-i \pi / 4}+\left(A_{1} \cos \theta\right.\right. \\
& \left.\left.+B_{1} \sin \theta\right) e^{-i 3 \pi / 4}\right],
\end{aligned}
$$

and the time variation of the far field potential can be obtained by taking the inverse Fourier transform of Eq. (9) with respect to time, bearing in mind that $\omega$ is kept positive in $\phi_{0}(\omega)$. It can then be shown ${ }^{26}$ that for the observer time $t$ $>r / c$,

$$
\left[\frac{1}{\sqrt{t-r / c}}\right]^{F}=e^{i(\omega r / c+\pi / 4)} \sqrt{\frac{\pi}{\omega}},
$$

and the inverse transform of Eq. (9) takes the form of a convolution integral, which is

$$
\begin{aligned}
\phi_{0}(r, \theta, t)= & \frac{1}{\pi} \sqrt{\frac{c}{2 r}} \int_{-\infty}^{t-r / c}\left(\int_{-L / 2}^{L / 2} v d x+\frac{\cos \theta}{c} \frac{\partial}{\partial \tau} \int_{-L / 2}^{L / 2} v x d x\right. \\
& \left.+\frac{\sin \theta}{c} \frac{\partial}{\partial \tau} \int_{-L / 2}^{L / 2} v \eta d x\right) \frac{d \tau}{\sqrt{t-\tau-r / c}}
\end{aligned}
$$

where $\tau$ represents the near field time and $\tau<t-r / c$. The far field acoustic pressure, $p^{-}$, is

$$
\begin{aligned}
p^{-}= & -\rho \frac{\partial \phi_{0}}{\partial t} \\
= & -\frac{\rho}{\pi} \sqrt{\frac{c}{2 r}} \frac{\partial}{\partial t} \int_{-\infty}^{t-r / c}\left(\int_{-L / 2}^{L / 2} v d x+\frac{\cos \theta}{c} \frac{\partial}{\partial \tau} \int_{-L / 2}^{L / 2} v x d x\right. \\
& \left.+\frac{\sin \theta}{c} \frac{\partial}{\partial \tau} \int_{-L / 2}^{L / 2} v \eta d x\right) \frac{d \tau}{\sqrt{t-\tau-r / c}}
\end{aligned}
$$

Equation (12) shows that the sound radiated consists of a monopole and two dipoles. The monopole is originated from the total fluctuating fluid mass flow due to the boundary vibration. One can notice that for small $v / c$, the dipoles are very weak when compared to the monopole and can be neglected as in Filippi et al. $^{23}$

\section{2. $y / d \rightarrow+\infty$}

The potential at very large distance in this case is a combination of the vortex potential and the flexible boundary vibration potential [the reverse of Eq. (7)], which is,

$$
\begin{aligned}
\phi= & \frac{\Gamma}{2 \pi}\left[\tan ^{-1}\left(\frac{R \sin \theta-y_{o}}{R \cos \theta-x_{o}}\right)-\tan ^{-1}\left(\frac{R \sin \theta+y_{o}}{R \cos \theta-x_{o}}\right)\right] \\
& +\frac{1}{\pi} \int_{-L / 2}^{L / 2} v \log \sqrt{(R \cos \theta-x)^{2}+(R \sin \theta-\eta)^{2}} d x \\
\approx & -\frac{\Gamma y_{o}}{\pi R} \cos \theta+\frac{1}{\pi} \log R \int_{-L / 2}^{L / 2} v d x \\
& -\frac{1}{\pi R} \int_{-L / 2}^{L / 2} v(x \cos \theta+\eta \sin \theta) d x .
\end{aligned}
$$

Following the procedure in the previous section, the far field pressure, $p^{+}$, is

$$
\begin{aligned}
p^{+}= & \frac{\rho}{\pi} \sqrt{\frac{c}{2 r}} \frac{\partial}{\partial t} \int_{-\infty}^{t-r / c}\left[\int_{-L / 2}^{L / 2} v d x+\frac{\cos \theta}{c} \frac{\partial}{\partial \tau}\left(\int_{-L / 2}^{L / 2} v x d x\right.\right. \\
& \left.\left.+\Gamma y_{o}\right)+\frac{\sin \theta}{c} \frac{\partial}{\partial \tau} \int_{-L / 2}^{L / 2} v \eta d x\right] \frac{d \tau}{\sqrt{t-\tau-r / c}}
\end{aligned}
$$

An additional dipole due to the unsteady axial vortex force is created. Its strength may be comparable to that of the monopole as the vortex velocity can be large compared to $v$. This will be discussed later.

\section{RESULTS AND DISCUSSION}

In the foregoing discussion, all length scales are normalized by $d$. The time is normalized by $d / U_{i}$. The tension $T$, damping $D$, and the surface density $M$ are normalized by $\rho U_{i}^{2} d, \rho U_{i}$, and $\rho d$, respectively. The in vacuo wave speed along the flexible boundary is $c_{T}=\sqrt{T / M}$ such that this wave speed is also normalized by $U_{i}$. In the real scenario, $c_{T}$ $=0.1 \mathrm{c}$ is essentially true. ${ }^{27}$ The present analysis is always under the low Mach number condition as in a practical flow duct and $L / d$ is set to 2 in the present investigation. The structural damping effect is insignificant in practice. According to the setup of Frendi et al. ${ }^{28}$ and the low Mach number 


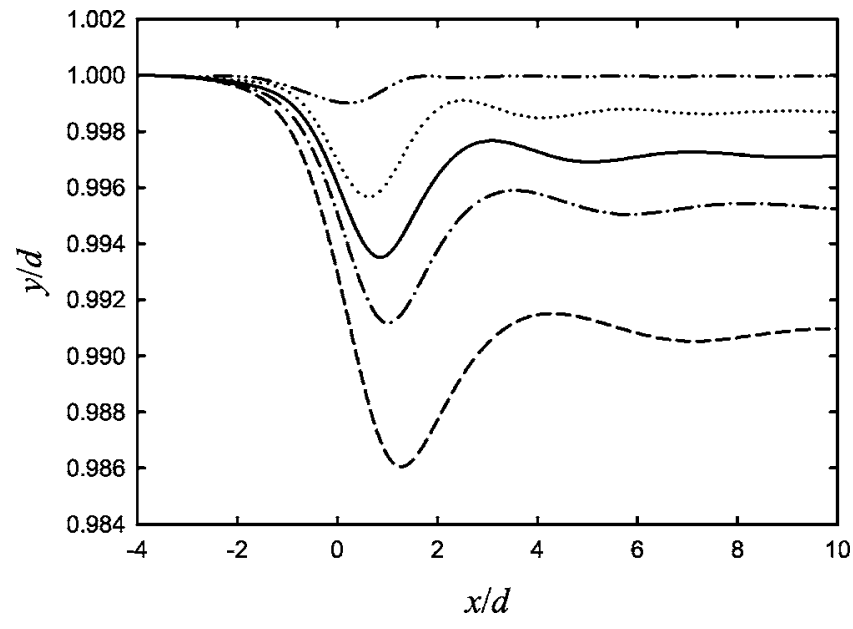

FIG. 2. Vortex flight path at $M=100$ : (--) $c_{T}=2 ;(\cdots) c_{T}=\sqrt{3 / 2} ;(-) c_{T}$ $=1 ;(--) c_{T}=\sqrt{3 / 4} ;(---) c_{T}=\sqrt{1 / 2}$.

condition invoked in the present study, $D$ ranges from 0.5 to 1 . Therefore, $D$ is set to unity in the present study.

The flexible boundary is discretized into 100 equal elements and it has been confirmed that further increase in this number does not produce any significant change to the computed results. Without loss of generality, the vortex is introduced initially at $x / d=-5$ and the corresponding vortex speed is closed to unity. An increase in this initial vortex distance from the flexible boundary does not have any significant effect on the results.

\section{A. Vortex and flexible boundary motion}

Figure 2 shows that effects of $c_{T}$ on the vortex trajectory at $M=100$. The vortex moves toward the flexible boundary for the range of $c_{T}$ tested. This agrees with the results of Tang $^{29}$ for a vortex moving over a surface with flow impedance. The transverse fluctuating vortex motion is due to the vibration of the flexible boundary which is absent in Tang. ${ }^{29}$ The vortex gets closer to the wall as it continues its path downstream of the flexible boundary. This appears to be inline with the results of Walker et al. ${ }^{24}$ though the vortex is kept moving with a constant speed in their study.

It is observed that the deviation of the vortex path from $y / d=1$ is small at large $c_{T}$. For $c_{T} \geqslant 2$, the vortex returns to its initial height above the rigid wall after flying over the flexible wall. For $c_{T}>1$, the vortex path varies slowly with increasing wave speed. The movement of the vortex path toward the flexible boundary increases rapidly for a relatively strong vortex $\left(c_{T}<1\right)$. The amplitude of the boundary vibration also increases with decreasing $c_{T}$, but it is observed to be below $5 \%$ of $d$ throughout the present investigation. This will be discussed further later. The corresponding trends for other values of $M$ are very similar to that shown in Fig. 2 provided that the maximum boundary vibration amplitude, $|\eta|_{\max }$, is small compared to $d$ and thus are not presented. However, one can expect that a higher $c_{T}$ is required to keep $|\eta|_{\max }$ small enough for the present model to apply when the surface density $M$ is reduced. For instance, when $M$ is close to 2 , which is the value adopted by Huang et al. ${ }^{27} T$ must be larger than 30 , indicating $c_{T}>3.87$ and a relatively weak

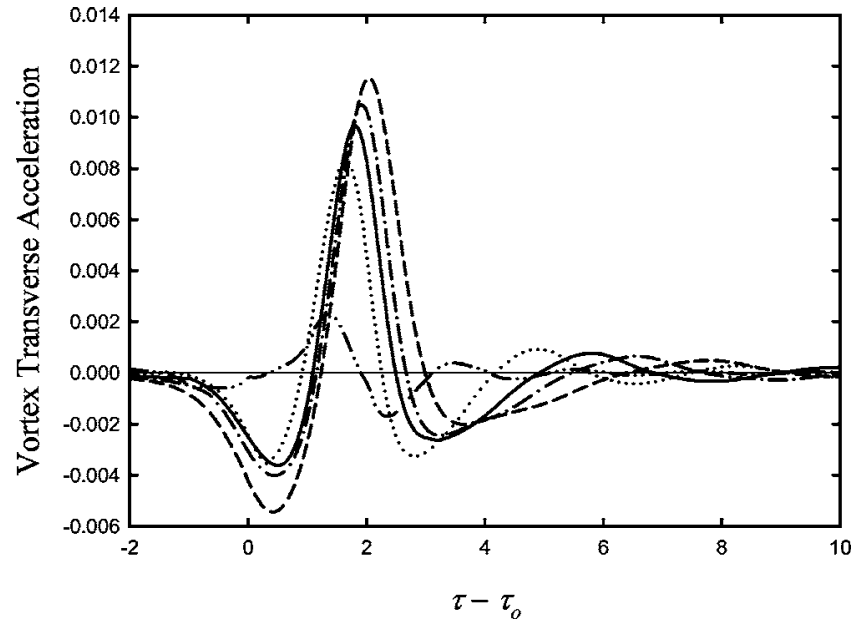

FIG. 3. Time variations of transverse velocity of vortex at $M=100$. Legends: same as those in Fig. 2.

vortex is required. However, such condition guarantees a very low Mach number flow and thus is still valid for air conditioning noise control application.

The transverse accelerations of the vortex with $M=100$ and different $c_{T}$ are shown in Fig. 3, where $\tau_{0}$ denotes the instant at which the vortex moves above the leading edge of the flexible boundary $(x=-L / 2)$. The amplitude of the corresponding axial components of the vortex accelerations are comparable to those shown in Fig. 3 and are more prevailing than the transverse accelerations. However, they are not presented because they do not have any direct contributions to the radiated sound field [Eq. (14)].

At lower $T$ (and thus lower $c_{T}$ ), the transverse acceleration of the vortex is more pulse-like. This is expected from the characteristics of the vortex paths shown in Fig. 2. A weaker $T$ results in a larger deflection of the flexible boundary and a relatively more pronounced effect of the edge at $x=-L / 2$, resulting in a faster downward movement of the vortex. Stronger sound scattering is expected but the very directional backward sound radiation by a semi-infinite rigid edge (for instance in Crighton ${ }^{21}$ ) does not exist in the present study. This is due to the very small vertical vibration of the flexible boundary at its edges so that the sharp edge effect is much attenuated as shown in one of the examples in Tang and Lau. ${ }^{20}$

Figure 4(a) shows the time variation of the flexible boundary displacement $\eta$ at $c_{T}=2, M=100$. The vortex is at $x / d=1$ (at the downstream edge of the boundary) at $\tau-\tau_{0}$ $\sim 2$. A relatively larger displacement $\left(|\eta|_{\max } \sim 0.5 \%\right.$ of $\left.d\right)$ is found when the vortex is moving over this boundary and a more periodical boundary motion is observed as the vortex propagates away from it. The corresponding vibration velocity of the flexible boundary is given in Fig. 4(b). While its magnitude is comparable or slightly higher than the transverse velocity of the vortex, its maximum is still $\sim 0.5 \%$ of the instantaneous vortex speed.

Another example of the flexible boundary vibration at $c_{T}=0.7071$ and $M=100$ is given in Fig. 5. The weaker tension within the boundary and thus a relatively stronger vortex results in a larger $\eta$, where the maximum amplitude is 


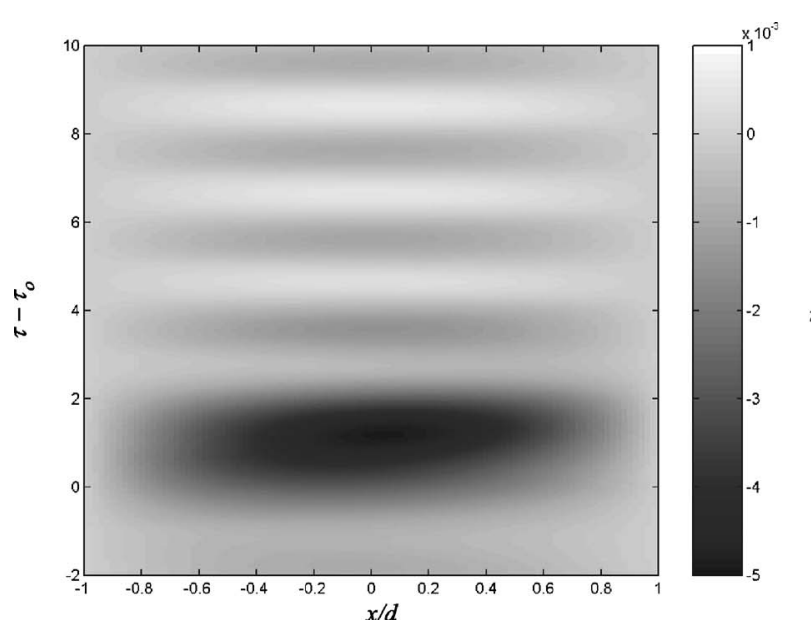

(a)

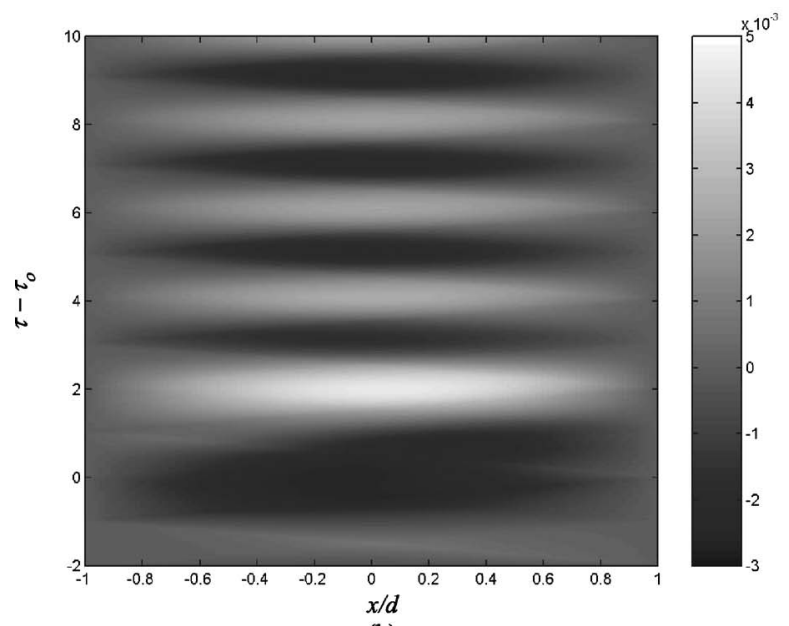

(b)

FIG. 4. Vibration pattern of the flexible boundary at $M=100$ and $c_{T}=2$. (a) Displacement; (b) velocity.

close to $5 \%$ of $d$. Again, the vortex is at $x / d=1$ at $\tau-\tau_{0}$ $\sim 2$ as the axial velocity of the vortex is not much affected by the flexible boundary. The vibration of the boundary is periodic. The pattern of boundary vibration velocity is not symmetrical about the central plane of this boundary [Fig. $5(\mathrm{~b})]$. The maximum boundary vibration velocity reaches $\sim 6 \%$ of the vortex speed as shown in Fig. 5(b).

\section{B. Far field acoustic radiation}

Since $c_{T}$ can be chosen to be $\sim 0.1 c$ as discussed in Huang et al. ${ }^{27} c_{T}=0.1 c$ is set throughout this section. However, it will be discussed later that the foregoing conclusions will not be affected by this assumption as far as the Mach number of the vortex motion remains low. The time integral of Eqs. (12) and (14) can be carried out numerically by a proper argument substitution as in Tang and Ko. ${ }^{30}$

At $y \rightarrow \infty$, the monopole radiation due to the boundary vibration dominates the radiated sound field as shown in Fig. 6(a) for a relatively weak vortex at $c_{T}=2(T=400, M=100)$ and thus $c=20$. The strength of the longitudinal vibration dipole is weak compared to that of the monopole, while that of the transverse vibration dipole is even much weaker and is not presented. The contribution from the dipole directly created by the transverse acceleration of the vortex (vortex di-

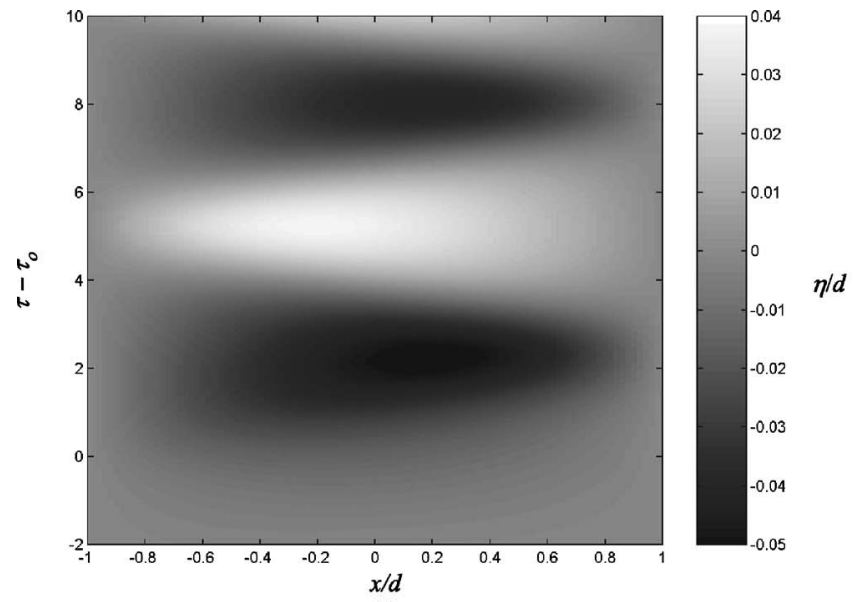

(a)

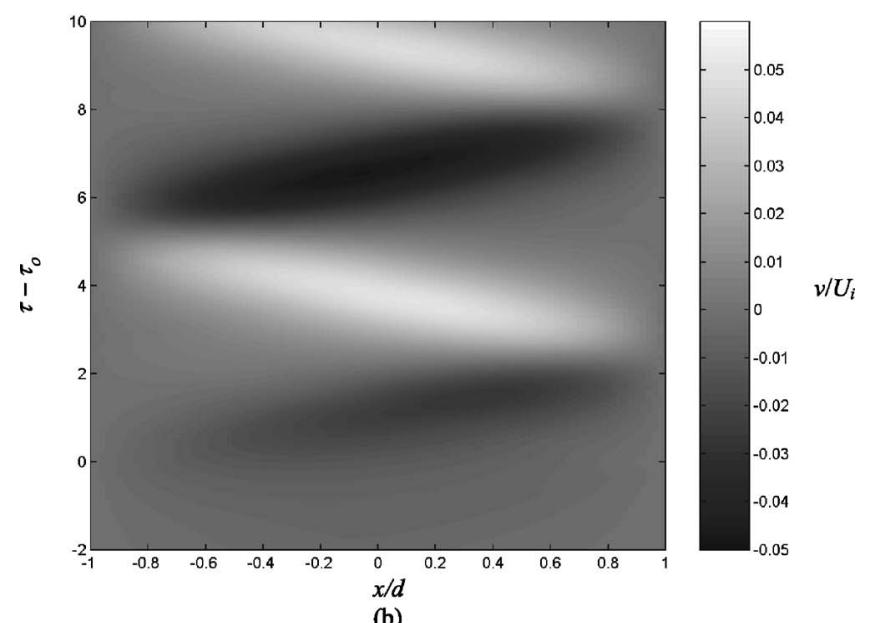

(b)

FIG. 5. Vibration pattern of the flexible boundary at $M=100$ and $c_{T}$ $=\sqrt{1 / 2}$. (a) Displacement; (b) velocity.

pole), which is pulse-like, is also weak in this case. However, it is stronger than the dipoles created directly by the boundary vibration when the vortex is in the proximity of the boundary.

The monopole is in-phase with the vortex dipole at $\theta$ $<90^{\circ}$ but the opposite is true at $\theta>90^{\circ}$. This gives rise to a slightly downstream-biased radiation, especially when the vortex is close to the downstream edge of the flexible boundary $\left(\tau-\tau_{0} \sim 2\right)$. This appears to be in-line with existing results of a low Mach number flow over a cavity where similar downstream-biased overall sound radiation is observed. ${ }^{31}$ Uncoupled model, such as that of Walker et al., ${ }^{24}$ which assumes no transverse vortex acceleration, appears to be insufficient for the study of flow-induced acoustic radiation. The increase in $c_{T}$ (and thus $c$ ) implies a lower flow Mach number and results in a reduction of the magnitude of the vortex dipole relative to that of the monopole. One should note that the radiation due to boundary vibration at $y \rightarrow-\infty$ is the reverse of that at $y \rightarrow \infty$ with the vortex dipole excluded.

Figure 6(b) shows the strengths of the monopole and the various dipoles at $c_{T}=1 / \sqrt{ } 2(c \sim 7.1), T=50$, and $M=100$. A reduction in $c_{T}$ increases the overall contribution of the vortex dipole and thus the extent of the downstream biased sound radiation. The strength of the vortex dipole is compa- 

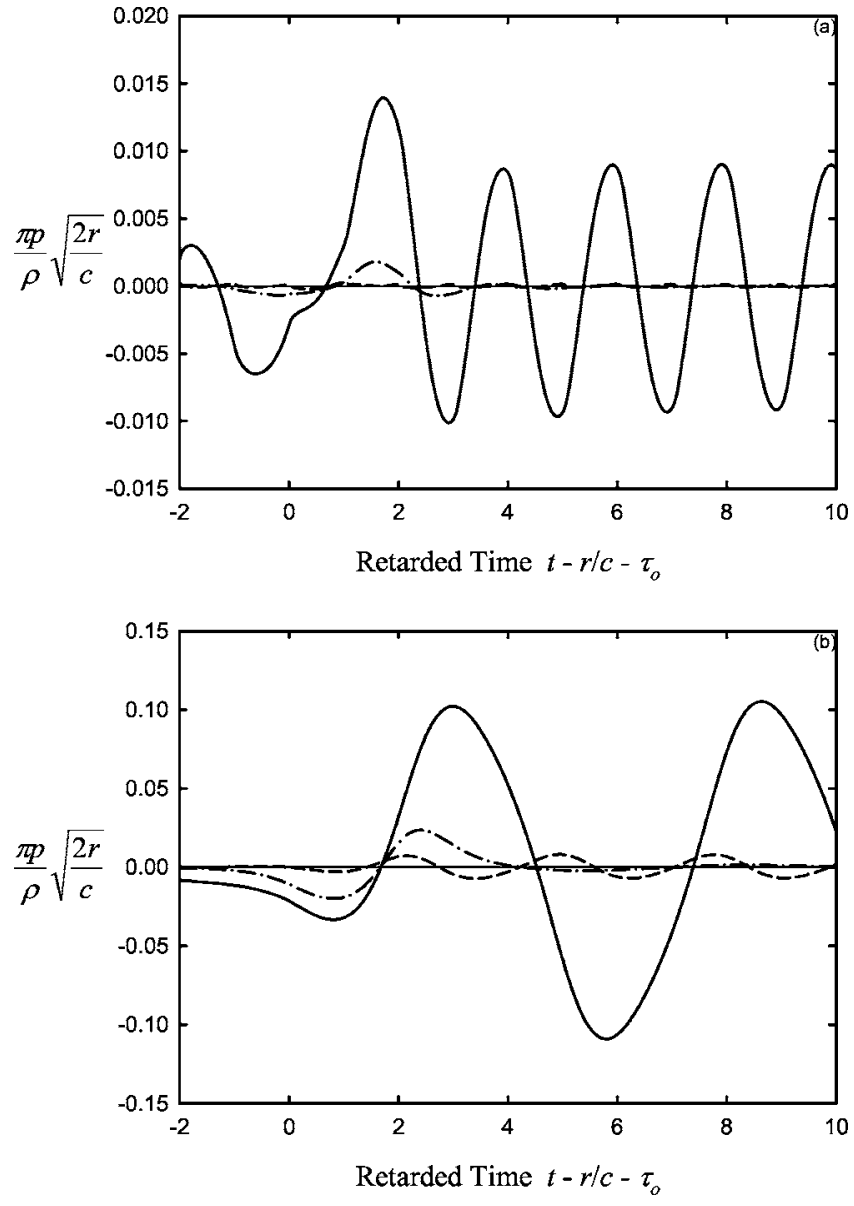

FIG. 6. Time fluctuations of sound fields' magnitudes at $M=100$. (a) $T$ =400; (b) $T=50$. (-) Monople; (-.-) vortex dipole; (---) longitudinal vibration dipole.

rable to that of the monopole when the vortex is flying over the flexible boundary, leading to weak upstream radiation. The longitudinal vibration dipole becomes more important, but is still weak when compared to the monopole and the vortex dipole. It can also be noticed that the period of the vibration dipole is shorter than that of the monopole. The transverse vibration dipole remains negligible. A stronger vortex leads to stronger acoustic radiation.

The evolution of the radiation directivity of the stronger vortex case $[$ Fig. $6(\mathrm{~b})]$ when the vortex is in close proximity of the flexible boundary is summarized in Fig. 7. The data at $\theta>180^{\circ}$ are those from the side of the boundary without the vortex. The weak longitudinal vibration dipole is capable of producing a slightly biased radiation at $\theta>180^{\circ}$, but the bias direction is continuously swapping between downstream and upstream depending on the signs of the dipole and the monopole (whose frequencies are not the same) until the strength of the dipole becomes negligible. For $\theta<180^{\circ}$, the stronger vortex dipole dominates the dipole field and creates a downstream biased sound radiation. Similar trends are observed for other values of $M$ (these results are not presented here).

The acoustic powers radiated on the two sides of the boundary are nearly the same at high $c_{T}$ due to the very weak contributions from the dipoles. At lower $c_{T}$, the contribution from the vortex dipole is restricted at the instant of intensive interaction between the vortex and the flexible boundary, but

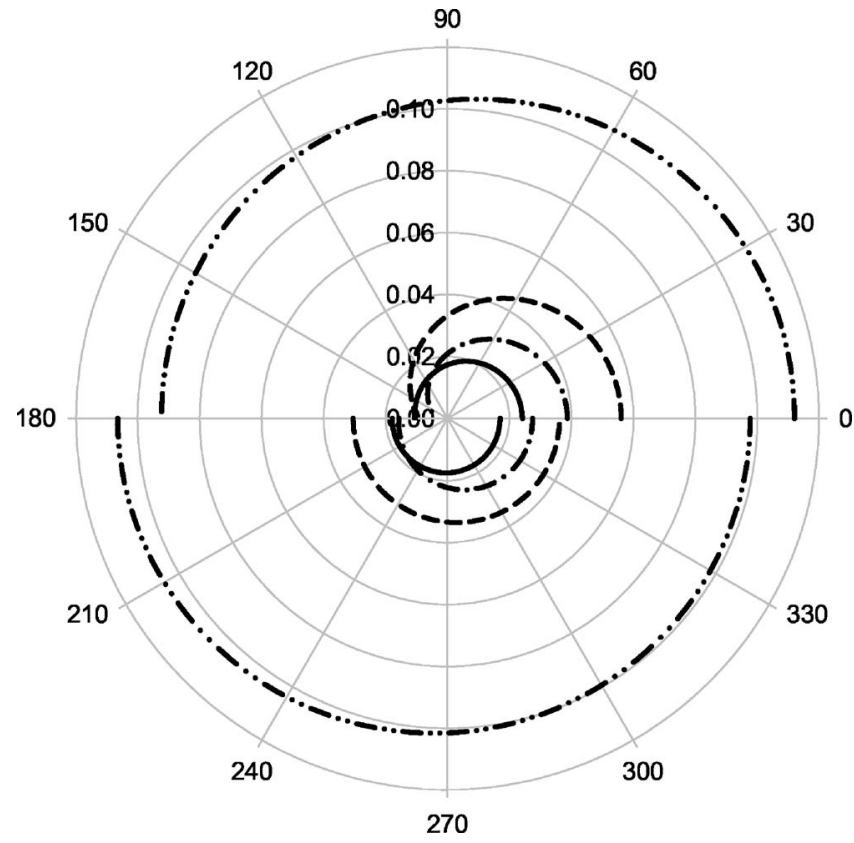

FIG. 7. Evolution of radiation directivity at $M=100$ and $T=50$. (-) $\tau-\tau_{0}$ $=-0.28 ;(---) \tau-\tau_{0}=0.81 ;(---) \tau-\tau_{0}=1.90 ;(-\cdot-) \tau-\tau_{0}=2.99$.

is still not very significant. Figure 8 illustrates the time variation of the "per unit spanwise length" acoustic power radiated at $y>0$, denoted by $W$ hereinafter, at various $c_{T}$ with $M=100$. In general, the acoustic power increases rapidly when the vortex is moving over the downstream edge of the flexible boundary and then reaches a maximum during the next cycle of boundary vibration before it is being damped down gradually due to structural damping and fluid loading. It is only at very high $c_{T}$ that the acoustic power is reduced quickly after the vortex moves away from the flexible boundary. However, the acoustic power in this case is very weak compared to those of the others presented in Fig. 8.

The maximum $W$ depends also on $M$ as shown in Fig. 9. A lighter boundary produces stronger acoustic radiation mainly in the form of monopole even the wave speed relative to the initial vortex speed is kept fixed (a weaker $T$ ). It

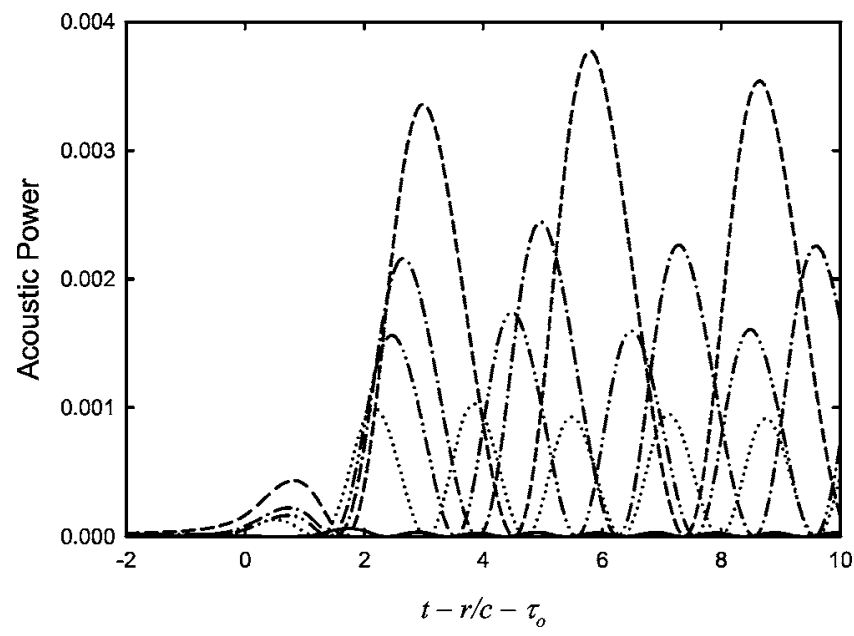

FIG. 8. Time variation of acoustic power radiated to upper side of flexible boundary at $M=100$. (-) $c_{T}=2 ;(\cdots) c_{T}=\sqrt{3 / 2} ;(-\cdot) c_{T}=1 ;(-\cdot-) c_{T}=\sqrt{3 / 4}$; (--) $c_{T}=\sqrt{1 / 2}$. 


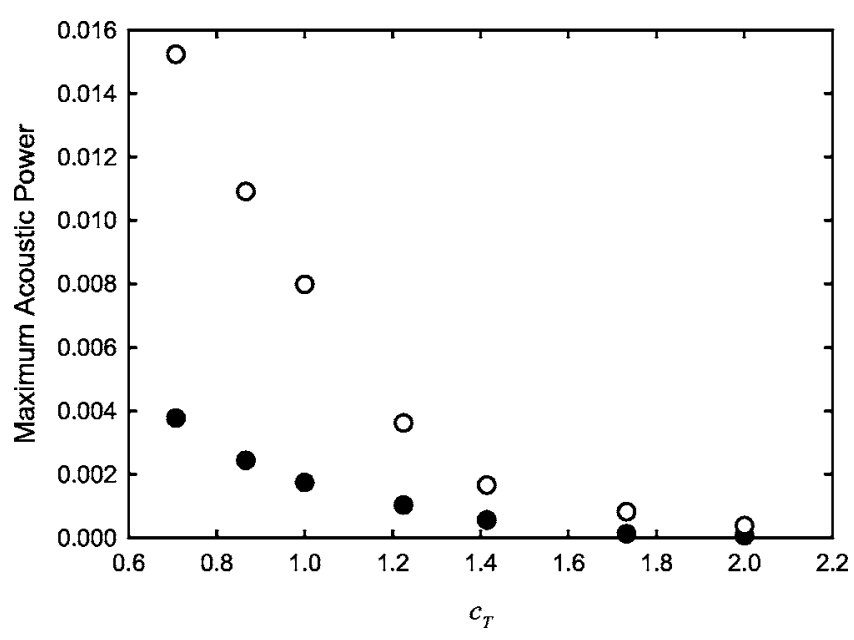

FIG. 9. Variation of maximum acoustic power radiated with $c_{T}$. (๑) $M$ =100; (○) $M=50$.

should be noted that $|\eta|_{\max }$ for the case of $M=50$ and $c_{T}$ $=0.7071$ reaches $10 \%$ of $d$, which exceeds the prescribed limit of the present study of $5 \%$. Nevertheless, the reduction of $M$ from 100 to 50 at fixed $c_{T}$ and $c$ in general results in 1.8 to 2.2 times increase in both $|\eta|_{\max },|v|_{\max }$ and the acceleration (not presented here). The radiated acoustic power tends to vary with $|v|_{\max }$ to a power of $\sim 2$ when $c_{T}$ is fixed. However, this power law index shows a tendency to increase when $c_{T}>1$, suggesting a faster reduction of the acoustical radiation efficiency as the vortex speed Mach number approaches the vanishing value.

For the two values of $M$ studied, the variation of maximum $W$ with $|v|_{\max }$ appears to follow a power law as shown in Fig. 10. The approximated relationship is

$$
W_{\max } \sim|v|_{\max }^{1.66} / \sqrt{M},
$$

with the result at $M=50$ and $c_{T}=0.7071$ falls slightly offtrend probably due to the relatively large boundary deflec-

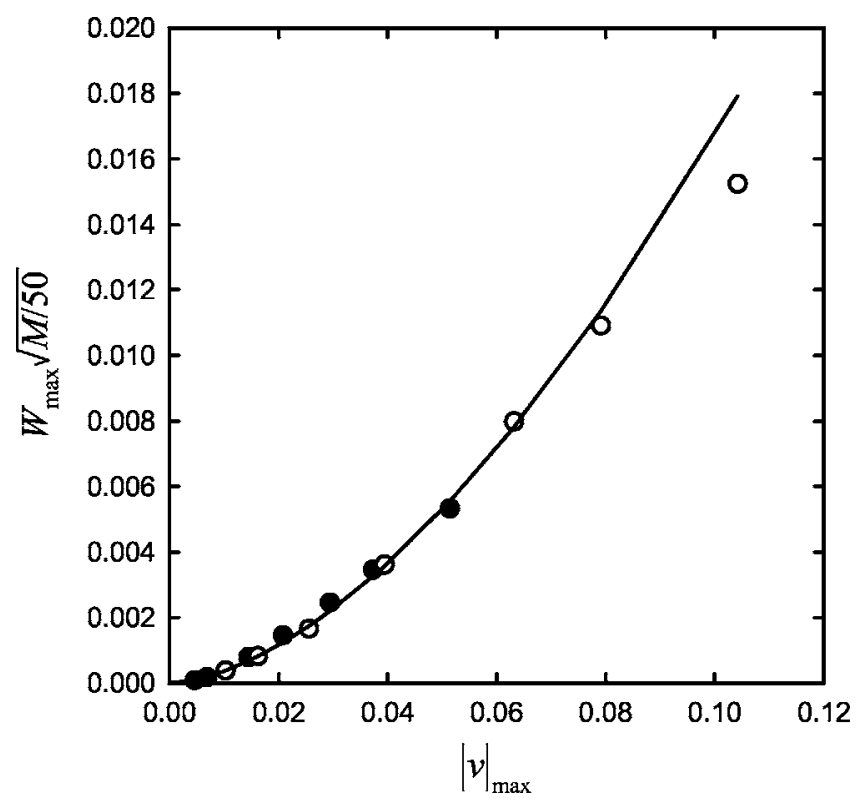

FIG. 10. Power law relations between vibration velocity and acoustic power radiation. (O) $M=100$; (○) $M=50 ;(-)$ power law curve [Eq. (15)]. tion. The power index is less than that for the compact harmonic monopole radiation, which is 2 (e.g., Kinsler et $\left.a l . .^{32}\right)$. This is common for two-dimensional radiation where the sound source is noncompact in its spanwise direction. It is expected that $W$ will scale on the overall volume flow induced by the boundary vibration through a similar power law relationship.

In the present study, the vortex moves under the influence of solid boundaries in the absence of a mean flow. However, it is believed that the presence of a low Mach number mean flow will tend to increase the acoustic power radiation as shown in Ffowcs Williams and Lovely, ${ }^{12}$ though their study is for a compact vibrating source. Such increase is expected to be more significant in the two-dimensional case as the corresponding amplification of acoustic radiation will then depend on the Mach number to a lower order. ${ }^{33}$

\section{CONCLUSIONS}

An inviscid model was developed to investigate the sound radiated due to the unsteady interactions between a vortex and a finite length flexible boundary in an otherwise rigid wall at low Mach numbers. The vibration displacement of the latter was kept small. The motion of the vortex was obtained basically through the potential theory and the sound radiated on the two sides of the flexible boundary was estimated using the matched asymptotic expansion technique.

The vortex path is deflected toward the flexible boundary and the deflection is larger as the tension in the boundary decreases. This results in larger boundary vibration magnitude and higher vortex transverse acceleration. The vortex continues its motion parallel to the rigid wall as time goes on, but at a lower height above the wall unless the wave speed along the flexible boundary is considerably higher than the initial vortex speed, which is the case for a relatively weak vortex.

The results of the matched asymptotic expansion show clearly that the rate of change of volumetric flow induced by the flexible boundary vibration and the transverse vortex acceleration are two major sources of sound. The former creates a monopole field and dominates the overall acoustic power radiation. The transverse vortex acceleration gives rise to a longitudinal dipole which is only significant to the acoustic radiation above the flexible boundary when the vortex is flying over this boundary. The interaction between these two sound fields results in a downstream-biased acoustic radiation.

The boundary vibration also creates one longitudinal and one transverse dipole. The latter is too weak to be important. The longitudinal dipole becomes a bit more important when the wave speed of the flexible boundary is reduced. This results in a slight bias in the acoustic radiation direction on the side of the boundary without the vortex. The radiation directivity swaps between downstream and upstream as the period of the longitudinal dipole strength variation is about double that of the monopole. It is also found that the strength of the per unit spanwise length acoustic power radiation scales approximately with the vibration-induced volumetric 
flow to a power of $\sim 1.66$ and the inverse of the square root of the surface density of the flexible boundary.

\section{ACKNOWLEDGMENT}

Funding support under Grant Number PolyU1/02C from the Research Grants Council of the Government of the HKSAR is gratefully acknowledged.

${ }^{1}$ A. Frendi and J. Robinson, "Effect of acoustic coupling on random and harmonic plate vibrations," AIAA J. 31, 1992-1997 (1993).

${ }^{2}$ A. Cummings, "Low frequency acoustic transmission through the walls of rectangular ducts," J. Sound Vib. 61, 327-345 (1978).

${ }^{3}$ L. Huang, "A theoretical study of duct noise control by flexible panels," J. Acoust. Soc. Am. 106, 1801-1809 (1999).

${ }^{4}$ W. K. Blake, "The acoustic radiation from unbaffled strips with application to a class of radiating panels," J. Sound Vib. 39, 77-103 (1975).

${ }^{5}$ F. G. Leppington, E. G. Broadbent, and K. H. Heron, "The acoustic radiation efficiency of rectangular panels," Proc. R. Soc. London, Ser. A 382, 245-271 (1982).

${ }^{6}$ S. F. Wu and L. Maestrello, "Responses of finite baffled plate to turbulent flow excitations," AIAA J. 33, 13-19 (1995).

${ }^{7}$ E. H. Dowell, "Radiation from panels as a source of airframe noise," AIAA J. 13, 1529-1530 (1975).

${ }^{8} \mathrm{M}$. S. Howe, "Diffraction radiation produced by turbulent boundary layer excitation of a panel," J. Sound Vib. 121, 47-65 (1988).

${ }^{9}$ I. D. Abrahams, "Scattering of sound by a finite non-linear elastic plate bounding a nearly resonant cavity," J. Sound Vib. 130, 387-404 (1989).

${ }^{10}$ F. G. Leppington, "Acoustic scattering by membranes and plates with line constraints," J. Sound Vib. 58, 319-332 (1978).

${ }^{11}$ W. R. Graham, "The influence of curvature on the sound radiated by vibrating panels,” J. Acoust. Soc. Am. 98, 1581-1595 (1995).

${ }^{12}$ J. E. Ffowes Williams and D. J. Lovely, "Sound radiation into uniformly flowing fluid by compact surface vibration," J. Fluid Mech. 71, 689-700 (1975).

${ }^{13}$ H. G. Davies, "Sound from turbulent boundary layer excited panels," J. Acoust. Soc. Am. 49, 878-889 (1971).

${ }^{14}$ L. L. Beranek, Noise and Vibration Control Engineering, Principles and Applications (Wiley, New York, 1992).

${ }^{15}$ M. J. Lighthill, "On sound generated aerodynamically I. General theory,'
Proc. R. Soc. London, Ser. A 211, 564-587 (1952).

${ }^{16}$ N. Curle, "The influence of solid boundaries upon aerodynamic sound," Proc. R. Soc. London, Ser. A 231, 505-514 (1955).

${ }^{17}$ M. S. Howe, Theory of Vortex Sound (Cambridge University Press, Cambridge, 2003).

${ }^{18} \mathrm{~F}$. Obermeier, "On a new representation of aeroacoustic source distribution. II. Two-dimensional model flows," Acustica 42, 62-71 (1979).

${ }^{19}$ R. C. K. Leung and R. M. C. So, "Noise generation of blade-vortex resonance," J. Sound Vib. 245, 217-237 (2001).

${ }^{20}$ S. K. Tang and C. K. Lau, "Vortex sound in the presence of a wedge with inhomogeneous surface flow impedance," J. Sound Vib. 281, 1077-1091 (2005).

${ }^{21}$ D. G. Crighton, "Basic principles of aerodynamic noise generation," Prog. Aerosp. Sci. 16, 31-96 (1975).

${ }^{22}$ H. R. Vallentine, Applied Hydrodynamics (Butterworth, London, 1969).

${ }^{23}$ P. J. T. Filippi, O. Lagarrigue, and P.-O. Mattei, "Perturbation method for sound radiation by a vibrating plate in a light fluid: Comparison with the exact solution," J. Sound Vib. 177, 259-275 (1994).

${ }^{24}$ J. D. A. Walker, C. N. Zhikharev, and T. J. Delph, "Induced oscillations of a finite plate," J. Guid. Control Dyn. 20, 1172-1180 (1997).

${ }^{25}$ P. M. Morse and K. U. Ingard, Theoretical Acoustics (McGraw-Hill, New York, 1968).

${ }^{26}$ I. S. Gradshteyn, I. M. Ryzhik, and A. Jeffrey, Tables of Integrals, Series and Products (Academic, Boston, 1994).

${ }^{27}$ L. Huang, Y. S. Choy, R. M. C. So, and T. L. Chong, "Experimental study of sound propagation in a flexible duct," J. Acoust. Soc. Am. 108, 624631 (2000).

${ }^{28}$ A. Frendi, L. Maestrello, and A. Bayliss, "Coupling between plate vibration and acoustic radiation," J. Sound Vib. 177, 207-226 (1994).

${ }^{29}$ S. K. Tang, "Effects of porous boundaries on the dynamics of an inviscid vortex filament," Q. J. Mech. Appl. Math. 54, 65-84 (2001).

${ }^{30}$ S. K. Tang and N. W. M. Ko, "Sound generation by interaction of two inviscid two-dimensional vortices," J. Acoust. Soc. Am. 102, 1463-1473 (1997).

${ }^{31}$ M. S. Howe, "Mechanism of sound generation by low Mach number flow over a wall cavity," J. Sound Vib. 273, 103-123 (2004).

${ }^{32}$ L. E. Kinsler, A. R. Frey, A. B. Coppens, and J. V. Sanders, Fundamentals of Acoustics, 4th ed. (Wiley, New York, 2000).

${ }^{33}$ J. E. Ffowcs Williams and D. L. Hawkings, "Shallow water wave generation by unsteady flow," J. Fluid Mech. 31, 779-788 (1968). 